\title{
Climate Change and Emerging Viral Diseases: the Evidence
}

\author{
Derek Gatherer \\ MRC-University of Glasgow Centre for Virus Research \\ Institute of Inflammation, Infection \& Immunity \\ College of Medical, Veterinary and Life Sciences \\ 8 Church Street \\ Glasgow G11 5JR \\ derek.gatherer@glasgow.ac.uk \\ (now: d.gatherer@lancaster.ac.uk)
}

\begin{abstract}
Three decades have now passed since the first papers linking climate change to issues in human disease and healthcare. One of the most active topics in this area has been the implication of climate change events, particularly temperature and humidity fluctuations, in the northward spread of vector-borne viruses from more tropical regions into Europe and North America. However, some detailed studies of one such emerging disease, tick-borne encephalitis virus (TBEv), have called the connection into question, concentrating the debate on the investigation of precise mechanisms for the spread of viral disease. More recently, firmer statistical correlations have been made between climate variables, the presence of insect vectors and the prevalence of viral disease, particularly for West Nile Virus (WNV). These insights suggest avenues for mechanistic confirmation of the involvement of climate change in other diseases where the connection remains conjectural.
\end{abstract}

The connection between weather, illness and mortality has a history extending back to Hippocrates ${ }^{1}$. Extremes of temperature appear to be a particular risk factor ${ }^{2}$. As awareness rose of potential global warming, the possibility of northward extension in the range of vector-borne pathogens was suggested, with particular focus on malaria and the rickettsial disease Rocky Mountain spotted fever, spread by mosquitoes and Dermacentor ticks respectively ${ }^{3}$. Similar suggestions were also made for the Aedes mosquito-borne flaviviral diseases yellow fever and dengue fever ${ }^{4}$, based around the observation that the northern limit of Aedes aegypti in the USA is at $35^{\circ} \mathrm{N}$ (equivalent to the southern border of the state of Tennessee), and of Aedes albopictus at $42^{\circ} \mathrm{N}$ (equivalent to the northern border of the state of California). A. albopictus has subsequently expanded its range slightly, now being found in some parts of Minnesota (at approx. $44^{\circ} \mathrm{N}$ ) and has even been reported in the Netherlands (at 
approx. $\left.52^{\circ} \mathrm{N}\right)$. However, this species is classified by the Invasive Species Specialist Group (http://www.issg.org/) as generally invasive and has spread to many countries in recent years. The involvement of climate change in this global colonization is as yet unclear.

Keywords: virology; emerging viruses; vector-borne diseases; climate change

\section{Classic case studies}

Such theoretical predictions were soon supplemented with actual examples. Six years of drought in the southwestern USA, followed by extremely heavy rain in the spring of 1993 was succeeded by a 10 -fold increase in the numbers of Peromyscus deer mice. The subsequent occurrence of an epidemic of Hantavirus pulmonary syndrome due to Sin Nombre virus ${ }^{5}$, of which Peromyscus is known to be the reservoir host ${ }^{6}$, provided a highly suggestive connection between a climate event and an outbreak of a serious human viral disease $^{7}$. An outbreak of dengue fever at Taxco in Mexico in 1988 at an altitude of 1700 metres, outside of the endemic area, was preceded by an unusually warm and dry spring with Aedes aegypti vectors congregating at domestic water containers ${ }^{8}$, a behavioural phenomenon also reported in Australia ${ }^{9}$. This case illustrates some of the difficulties in interpreting this kind of data. The Aedes vector was endemic to the Taxco area, but dengue fever was not. The epidemic may have been due to the shortening of the extrinsic incubation period (i.e. when the virus is replicating within the vector) following raised average temperature, or to the vector coming into closer domestic contact with potential human hosts due to the dry conditions. Unfortunately, comparative data on mosquito density at water containers was not collected at the time. The mean monthly maximum temperature of $32^{\circ} \mathrm{C}$ at Taxco in April 1988 would have been just sufficient to shorten the extrinsic incubation period from 12 days to 7 days ${ }^{10}$. These observations were soon built into theoretical models correlating temperature with likelihood of dengue virus transmission which in some cases were consonant with historical data on dengue fever outbreaks and which predicted substantial increases in its incidence by the mid-to-late $21^{\text {st }}$ century $11-13$.

One of the most spectacular examples of range expansion is Bluetongue virus (BTV) which has spread into southern Europe and then even further northwards since $2006^{18}$. Initially spread by the midge Culicoides imola, it is now possibly spread by an unidentified vector, perhaps also a member of the genus Culicoides in areas 
north of $C$. imola's range. $C$. imicola was itself a recent arrival in Europe, its range expansion customarily attributed to climate change. However, the sudden outbreak of BTV serotype 8 in northwestern Europe in 2006 suggested that a direct introduction, via infected vertebrates or midges transported for business or accidentally into the region, was to blame. Sporadic outbreaks of BTV serotypes 6,9 and 11 have also occurred. BTV serotype 1 by contrast is expanding its range steadily northwards through France in a manner more typical of what would be predicted by the gradual climate change model.

As this paradigm was absorbed by the virological community, increasing numbers of viruses became the subject of similar predictions: orbiviruses ${ }^{14-18}$, phleboviruses $19-22$, hantaviruses ${ }^{23-25}$, avian influenza 26-28, arenaviruses ${ }^{29}$, bunyaviruses ${ }^{30}$, noroviruses ${ }^{31}$, alphaviruses $^{32}$ and flaviviruses ${ }^{32-35}$ including tick-borne encephalitis virus $^{36,37}$.

\section{The Onset of Revisionism}

By contrast, tick-borne encephalitis provided an interesting example of how climate change was predicted to restrict the range of a viral disease rather than increase it. However, it was also apparent that the disease was on the increase. This paradox led to a detailed reexamination of the whole hypothesis. The vector of tick-borne encephalitis virus (TBEv) is Ixodes ricinus which is found across Europe and neighbouring regions. However, TBEv is confined to Eastern Europe and the Baltic region, since its effective transmission is dependent on a precise pattern of seasonal temperatures, specifically warm enough for rapid tick egg development in summer with an autumn cooling to synchronize larval development in such a way that new uninfected larvae and infected tick nymphs from previous seasons are feeding simultaneously on rodent hosts by spring. Efficient transmission of the virus to the new batch of larvae is thus ensured. The ground must also be moist enough to ensure tick survival when not feeding on a host. A computer model combining these variables with current climatic data as input accurately predicts the current distribution of the virus ${ }^{36}$. Manipulating the input to simulate climate change towards hotter and drier summers predicts that the virus will lose most of its current range by 2050 and will have moved into an essentially new and greatly restricted habitat in southern Finland and some mountainous areas in central Europe that are currently not infected. TBEv is thus a candidate for a climate change-induced "un-emerging virus". Despite this, TBE cases have been increasing in central and eastern Europe since 
1993. In the former Warsaw Pact states, social changes involving increased travel to tick-infested forest areas (leisure for the new middle classes and foraging for the newly impoverished) and increased consumption of un-pasteurised milk from infected livestock, have been proposed. However, a similar rise has been seen in the former West Germany where these new social conditions do not apply ${ }^{36}$. As a result of these observations, further variables were proposed to be involved, for instance an increase in the density of roe deer, the main host of the adult ticks, immunization/screening programmes ${ }^{36,38}$ travel with pets ${ }^{37}$ or closure of collective farms with subsequent reforestation and reduction in pesticides and pollution ${ }^{39,40}$. Subsequent modelling led to the conclusion that climate change is possibly a minor factor among many ${ }^{38-41}$.

Such considerations led, from the early 2000s, to some general expressions of scepticism ${ }^{42,43}$. More stringent criteria began to be advanced for evidence of the effect of climate change on virus emergence. Among the sharper questions that must now be answered for each proposed incidence are:

1. Do the climate change data for the period under consideration demonstrate any significant trend? This is not the same thing as asking if there are long-term trends in global warming, merely an insistence that global warming is not used as a general explanation without regard to short-term fluctuations in temperature and precipitation etc.

2. If a statistically significant climatic alteration can be found for the period under consideration, does it apply to the geographical area where the emergence event is occurring?

3. For vector-borne diseases, does the emergence event coincide with any significant change in the distribution, behaviour or abundance of the known vector?

4. If so, is the change to the vector population applicable to the geographical area where the emergence event is occurring?

5. Do the changes in the vector population correspond to changes in the viral population? For instance, are the vectors carrying more virus?

6. If a temperature-related effect on virus propagation in the vector is proposed can that be demonstrated in the laboratory, or even better in field samples obtained at the time of the emergence event? 
If these factors can all be answered in the affirmative, there is a case for climate change as a precipitating event in emergence of a new disease. However, the following must also be considered:

1) Has the identified climate change event produced any other change in the behaviour of the human (or animal in the case of veterinary viruses) victims? For instance, does hot weather produce a behavioural adaptation that increases risk of exposure to the virus?

2) Is a novel vector involved?

3) What other changes have occurred in the environment? This is an immensely broad question and could include economic, social, demographic and technological factors.

The first of these would include, for instance, the use of domestic water tanks identified in Mexico and Australia as a possible confounding factor, or the increased use of forests by human identified in the TBEv cases in eastern Europe. The second of these has been found to be a complication in the study of the spread of Bluetongue virus in Europe, where the expansion of the range of the virus and that of its classic vector are not congruent. The third is possibly so broad a category that it could never be entirely excluded.

\section{Focussing on mechanisms}

Recent publications are far less likely to adopt a broad-brush attitude to the spread of erstwhile tropical diseases and the global warming trend. In this new mood of mechanism-oriented investigation, two principal approaches may be discerned:

1. those papers which take existing data demonstrating a correlation between established virus activity and some climatic variable and then extrapolate this to a future time when long-term climate change trends have been more fully worked out.

2. those papers which retrospectively analyse a virus emergence event against climatic data and attempt to connect them.

One salient example of the first approach has been some recent work on human seasonal influenza $A^{44}$. The existence of winter peaks in influenza cases, alternating in the northern and southern hemispheres, has been obvious for many years and is a classic of biometeorology, predating the current concern with longer-term 
climatic changes. Quite why influenza and other respiratory viruses are so much more prevalent in the winter has never been satisfactorily resolved, but phylogenetic studies have recently shown that seasonal influenza A does not retreat to local refugia in the summer, but rather spreads afresh from tropical regions of south-east Asia at the beginning of each winter season ${ }^{45}$. In the tropics, human influenza shows much weaker seasonality. In Hong Kong, detailed records of influenza A hospital admissions over a 10year period from 1997 to 2006 were compared with temperature and humidity records ${ }^{44}$. Influenza A demonstrated two annual peaks, in late winter and summer and its activity correlated in the climatic records with humid periods (monthly humidity averages between $60 \%$ and $90 \%$ ), when coupled with average daily temperature maxima (tending to $30^{\circ} \mathrm{C}$ ) and minima (down to $15^{\circ} \mathrm{C}$ ). Influenza B, by contrast, was only correlated with cold humid weather, consistent with it having a more pronounced late winter peak. However, despite the robust statistical correlation between influenza incidence and weather variables, several questions remain. For instance, average daily temperature maxima and minima did not change in Hong Kong over the period studied, so no correlation can be made between influenza incidence and long-term temperature change, as opposed to seasonal weather variation. However, from 2001 onwards, humidity was consistently lower than in the first half of the study decade. The years 1998, 2004, 2005 and 2006 were particularly severe years for influenza, but this observation is complicated by the fact that influenza will vary in intensity from year to year due to the unpredictable antigenic drift of the virus. Additionally, 2003 was a very low year for influenza incidence owing to the public health measures taken to control SARS, which incidentally appear to have halted other respiratory viruses. Nevertheless, based on the statistical correlations, the prediction was made that a $2^{\circ} \mathrm{C}$ rise in average daily temperature would lengthen the summer peak period for influenza $A$ and decrease the late winter peak periods for both influenza $A$ and $B$. The issue of the mechanism by which temperature and humidity affects influenza virus transmission, remains unresolved.

A similar study has recently been performed for dengue fever, which unlike influenza is an emerging virus colonizing a new geographical range ${ }^{46}$. In Thailand, part of dengue fever's original range, meteorological data were collated for 2005-2007 against dengue incidence. Warm and wet weather were found to be significantly correlated with dengue fever incidence and again used as a basis for extrapolation into a scenario of a long-term temperature rise of $1.75^{\circ} \mathrm{C}$, which would lengthen the dengue fever season from the current 5 months to 9 months. This study also found positive correlations between dengue fever incidence and 
social factors, including large numbers of children, low educational level and the outdoor water storages previously identified as risk factors for many other vector borne diseases. This suggests that social change could influence dengue fever incidence independently of any climatic factors.

However, it is in West Nile Virus (WNV) where the closest approach to the construction of a mechanistic chain linking virus emergence with climate change has been developed. For human WNV infections in Mississippi during 2002, the regions with the lowest rainfall in the previous year had the highest incidence of WNV in humans ${ }^{47}$. The Culex mosquitoes that spread WNV prefer dry weather in contrast to the Aedes mosquitoes spreading dengue fever. Computer models have been developed that predict host mosquito abundance from atmospheric and habitat variables and these have been verified experimentally against data from mosquito collections in the wild in both Florida and California ${ }^{48}$. During the warm winter and drought of early 2009 in California, in which some daily maximum temperatures reached $10^{\circ} \mathrm{C}$ above the seasonal norm, many locations had increased numbers of mosquitoes ${ }^{49}$. Several features were observed in the sample giving indications of how dry weather increases Culex numbers: quiescent mosquitoes resumed activity in the warmer conditions; the mosquito reproductive cycle was re-commenced earlier; there was lower mortality in overwintering adults. Interestingly, however, the swollen Californian Culex populations of early 2009 were apparently negative for WNV and two other arboviruses despite the fact that WNV normally appears in the study areas from April onwards. It therefore seems that conditions conducive to the expansion of the mosquito population do not necessarily lead to expansion of the virus population. A study in the Chicago areas covering the years 2004-2008 focussed on this issue of infection rate within the Culex vector, finding that $80 \%$ of the variation was accountable to prior weather conditions, and noting that a burst of wet weather following a dry period was a factor, possibly accounting for the absence of viruses in the 2009 Californian sample ${ }^{50}$.

One potential avenue of further research is in the laboratory. Ecosimulation cultures of bacteria with grazing nanoflagellates and infecting bacteriophages can be created and then exposed to increases in temperature and ultraviolet light ${ }^{51}$. However, the applicability of such bacterial systems to vector-borne mammalian viruses is clearly limited.

\section{Conclusions}


There are two extremes in the biometeorology of climate change. An uncritical acceptance of the reasonable proposal that the effects of climate change are bound to increase the prevalence of tropical diseases in previously temperate regions, versus the paralysing scepticism that demands full comprehension of every single variable in the system before blame can be laid at the foot of global warming. The first of these disregards the value of detailed investigation into virus emergence for the sake of a foregone conclusion and may sacrifice opportunities for a greater understanding of virus dynamics. The second renders research an impossible task, as complete data on all potential variables affecting a virus emergence event could never be collected.

1. Falagas, M., Bliziotis, I., Kosmidis, J. \& Daikos, G. Unusual climatic conditions and infectious diseases: observations made by Hippocrates. Enferm Infecc Microbiol Clin. 28, 716-718 (2010).

2. Kalkstein, L. \& Valimont, K. Climate effects on human health. in Potential effects of future climate changes on forests and vegetation, agriculture, water resources, and human health. EPA Science and Advisory Committee Monograph no. 25389 122-152 (U.S. Environmental Protection Agency, Washington, D.C., 1987).

3. Longstreth, J. Anticipated public health consequences of global climate change. Environ Health Perspect 96, 139-44 (1991).

4. Shope, R. Global climate change and infectious diseases. Environ Health Perspect 96, 171-4 (1991).

5. Chapman, L.E. \& Khabbaz, R.F. Etiology and epidemiology of the Four Corners hantavirus outbreak. Infect Agents Dis 3, 234-44 (1994).

6. Childs, J.E. et al. Serologic and genetic identification of Peromyscus maniculatus as the primary rodent reservoir for a new hantavirus in the southwestern United States. J Infect Dis 169, 1271-80 (1994).

7. Patz, J.A., Epstein, P.R., Burke, T.A. \& Balbus, J.M. Global climate change and emerging infectious diseases. Jama 275, 217-23 (1996).

8. Herrera-Basto, E., Prevots, D.R., Zarate, M.L., Silva, J.L. \& Sepulveda-Amor, J. First reported outbreak of classical dengue fever at 1,700 meters above sea level in Guerrero State, Mexico, June 1988. Am J Trop Med Hyg 46, 649-53 (1992).

9. Beebe, N.W., Cooper, R.D., Mottram, P. \& Sweeney, A.W. Australia's dengue risk driven by human adaptation to climate change. PLoS Negl Trop Dis 3, e429 (2009).

10. Watts, D.M., Burke, D.S., Harrison, B.A., Whitmire, R.E. \& Nisalak, A. Effect of temperature on the vector efficiency of Aedes aegypti for dengue 2 virus. Am J Trop Med Hyg 36, 143-52 (1987).

11. Jetten, T.H. \& Focks, D.A. Potential changes in the distribution of dengue transmission under climate warming. Am J Trop Med Hyg 57, 285-97 (1997).

12. Patz, J.A., Martens, W.J., Focks, D.A. \& Jetten, T.H. Dengue fever epidemic potential as projected by general circulation models of global climate change. Environ Health Perspect 106, 147-53 (1998). 
13. Hales, S., de Wet, N., Maindonald, J. \& Woodward, A. Potential effect of population and climate changes on global distribution of dengue fever: an empirical model. Lancet 360, 830-4 (2002).

14. Wittmann, E.J. \& Baylis, M. Climate change: effects on culicoides-transmitted viruses and implications for the UK. Vet J 160, 107-17 (2000).

15. Mellor, P.S. \& Hamblin, C. African horse sickness. Vet Res 35, 445-66 (2004).

16. Purse, B.V. et al. Modelling the distributions of Culicoides bluetongue virus vectors in Sicily in relation to satellite-derived climate variables. Med Vet Entomol 18, 90-101 (2004).

17. Wilson, A. \& Mellor, P. Bluetongue in Europe: vectors, epidemiology and climate change. Parasitol Res 103 Suppl 1, S69-77 (2008).

18. Maclachlan, N.J. \& Guthrie, A.J. Re-emergence of bluetongue, African horse sickness, and other orbivirus diseases. Vet Res 41, 35 (2010).

19. Bi, P., Zhang, Y. \& Parton, K.A. Weather variables and Japanese encephalitis in the metropolitan area of Jinan city, China. J Infect 55, 551-6 (2007).

20. Gould, E.A., Higgs, S., Buckley, A. \& Gritsun, T.S. Potential arbovirus emergence and implications for the United Kingdom. Emerg Infect Dis 12, 549-55 (2006).

21. Gale, P. et al. Assessing the impact of climate change on vector-borne viruses in the EU through the elicitation of expert opinion. Epidemiol Infect 138, 21425 (2010).

22. Collao, X. et al. Genetic diversity of Toscana virus. Emerg Infect Dis 15, 5747 (2009).

23. Tersago, K. et al. Hantavirus disease (nephropathia epidemica) in Belgium: effects of tree seed production and climate. Epidemiol Infect 137, 250-6 (2009).

24. Klempa, B. Hantaviruses and climate change. Clin Microbiol Infect 15, 518-23 (2009).

25. Dearing, M.D. \& Dizney, L. Ecology of hantavirus in a changing world. Ann N Y Acad Sci 1195, 99-112 (2010).

26. Gatherer, D. The Little Ice Age and the emergence of influenza A. Med Hypotheses 75, 359-62 (2010).

27. Gilbert, M., Slingenbergh, J. \& Xiao, X. Climate change and avian influenza. Rev Sci Tech 27, 459-66 (2008).

28. Vandegrift, K.J., Sokolow, S.H., Daszak, P. \& Kilpatrick, A.M. Ecology of avian influenza viruses in a changing world. Ann N Y Acad Sci 1195, 113-28 (2010).

29. Clegg, J.C. Influence of climate change on the incidence and impact of arenavirus diseases: a speculative assessment. Clin Microbiol Infect 15, 504-9 (2009).

30. Elliott, R.M. Bunyaviruses and climate change. Clin Microbiol Infect 15, 5107 (2009).

31. Rohayem, J. Norovirus seasonality and the potential impact of climate change. Clin Microbiol Infect 15, 524-7 (2009).

32. Russell, R.C. Mosquito-borne arboviruses in Australia: the current scene and implications of climate change for human health. Int J Parasitol 28, 955-69 (1998).

33. Brugger, K. \& Rubel, F. Simulation of climate-change scenarios to explain Usutu-virus dynamics in Austria. Prev Vet Med 88, 24-31 (2009). 
34. Epstein, P.R. West Nile virus and the climate. J Urban Health 78, 367-71 (2001).

35. Brault, A.C. Changing patterns of West Nile virus transmission: altered vector competence and host susceptibility. Vet Res 40, 43 (2009).

36. Randolph, S.E. \& Rogers, D.J. Fragile transmission cycles of tick-borne encephalitis virus may be disrupted by predicted climate change. Proc Biol Sci 267, 1741-4 (2000).

37. Beugnet, F. \& Marie, J.L. Emerging arthropod-borne diseases of companion animals in Europe. Vet Parasitol 163, 298-305 (2009).

38. Randolph, S.E. Evidence that climate change has caused 'emergence' of tickborne diseases in Europe? Int J Med Microbiol 293 Suppl 37, 5-15 (2004).

39. Sumilo, D. et al. Climate change cannot explain the upsurge of tick-borne encephalitis in the Baltics. PLoS One 2, e500 (2007).

40. Sumilo, D. et al. Behavioural responses to perceived risk of tick-borne encephalitis: vaccination and avoidance in the Baltics and Slovenia. Vaccine 26, 2580-8 (2008).

41. Randolph, S.E. Dynamics of tick-borne disease systems: minor role of recent climate change. Rev Sci Tech 27, 367-81 (2008).

42. Zell, R. Global climate change and the emergence/re-emergence of infectious diseases. Int J Med Microbiol 293 Suppl 37, 16-26 (2004).

43. Zell, R., Krumbholz, A. \& Wutzler, P. Impact of global warming on viral diseases: what is the evidence? Curr Opin Biotechnol 19, 652-60 (2008).

44. Chan, P.K. et al. Seasonal influenza activity in Hong Kong and its association with meteorological variations. J Med Virol 81, 1797-806 (2009).

45. Nelson, M.I., Simonsen, L., Viboud, C., Miller, M.A. \& Holmes, E.C. Phylogenetic analysis reveals the global migration of seasonal influenza A viruses. PLoS Pathog 3, 1220-8 (2007).

46. Sriprom, M., Chalvet-Monfray, K., Chaimane, T., Vongsawat, K. \& Bicout, D.J. Monthly district level risk of dengue occurrences in Sakon Nakhon Province, Thailand. Sci Total Environ 408, 5521-8 (2010).

47. Wang, G., Minnis, R.B., Belant, J.L. \& Wax, C.L. Dry weather induces outbreaks of human West Nile virus infections. BMC Infect Dis 10, 38 (2010).

48. Morin, C.W. \& Comrie, A.C. Modeled response of the West Nile virus vector Culex quinquefasciatus to changing climate using the dynamic mosquito simulation model. Int J Biometeorol 54, 517-29 (2010).

49. Reisen, W.K. et al. Effects of warm winter temperature on the abundance and gonotrophic activity of Culex (Diptera: Culicidae) in California. J Med Entomol 47, 230-7 (2010).

50. Ruiz, M.O. et al. Local impact of temperature and precipitation on West Nile virus infection in Culex species mosquitoes in northeast Illinois, USA. Parasit Vectors 3, 19 (2010).

51. Bouvy, M. et al. Trophic interactions between viruses, bacteria and nanoflagellates under various nutrient conditions and simulated climate change. Environ Microbiol (2011). 